\title{
CUBIC MnTe - GROWTH BY MOLECULAR BEAM EPITAXY AND BASIC STRUCTURAL CHARACTERIZATION
}

\author{
A.K. ZAKRZEWSKI ${ }^{a}$, E. JANIK ${ }^{a}$, E. DYNOWSKA ${ }^{a}$, M. LESZCZYŃSKI $^{b}$, \\ M. KUtrowsKi ${ }^{a}$, T. WOJTOWICZ ${ }^{a}$, G. KARCZEWSKI ${ }^{a}$, J. BAz-MISIUK $^{a}$, \\ J. Domagala ${ }^{a}$ AND J. KossuT ${ }^{a}$ \\ ${ }^{a}$ Institute of Physics, Polish Academy of Sciences \\ Al. Lotników 32/46, 02-668 Warszawa, Poland \\ ${ }^{b}$ Center for High Pressure Research, Polish Academy of Sciences \\ Sokołowska 29, 01-142 Warszawa, Poland
}

\begin{abstract}
We report on growth by molecular beam epitaxy of thick layers of $\mathrm{MnTe}$ with zinc blende structure. Films as thick as $5.6 \mu \mathrm{m}$ were obtained. Characterization by X-ray diffraction proved their good structural quality. We determined the lattice constant and its temperature dependence. Broad luminescence due to internal $\mathrm{Mn}^{2+}$ transitions was observed. It showed an unexpected temperature dependence.
\end{abstract}

PACS numbers: 75.50.Pp, 68.55.Bd, 68.55.Jk

\section{Introduction}

Bulk crystals of magnetic semiconductor MnTe obtained by equilibrium growth techniques crystallize in the hexagonal NiAs structure with lattice constants $a=4.15 \AA, c=6.72 \AA[1]$. The direct band gap of this form of MnTe is $1.3 \mathrm{eV}$ [2]. From the studies of ternary diluted magnetic semiconductors (DMS), such as $\mathrm{Cd}_{1-x} \mathrm{Mn}_{x} \mathrm{Te}, \mathrm{Zn}_{1-x} \mathrm{Mn}_{x} \mathrm{Te}$ or $\mathrm{Hg}_{1-x} \mathrm{Mn}_{x} \mathrm{Te}$, it was inferred that a hypothetical MnTe in zinc blende cubic form should possess a wide band gap equal to $\approx 3.5 \mathrm{eV}$ and the lattice constant equal to $6.34 \AA$ [3]. Recently cubic MnTe was obtained by nonequilibrium growth techniques: molecular beam epitaxy (MBE) [4] and ionized cluster beam deposition [5]. Cubic MnTe is very interesting, because it offers an opportunity to study a nearly isotropic magnetic system having cubic symmetry with both first- and second-neighbor interactions being antiferromagnetic and the first neighbors interacting by an order of magnitude stronger than the second neighbors. Also the energy gap of cubic MnTe is quite large. It is, thus, a natural component of short wavelength optoelectronic applications. 


\section{Growth}

Zinc blende MnTe epilayers were grown by MBE in EPI- 620 machine. The growth was performed on (100)-oriented epi-ready GaAs $2^{\circ}$-off substrates (Sumitomo). After desorption of oxides from the substrate we deposited a thin layer (of order of $100 \AA$ ) of $\mathrm{ZnTe}$ in order to facilitate a (100)-oriented growth. Then, we followed with a deposition of buffer layer of either $\mathrm{ZnTe}$ or CdTe of the thickness ranging from $0.5 \mu \mathrm{m}$ to $2 \mu \mathrm{m}$. The buffer layers were grown in order to reduce the lattice constant mismatch between the substrate and MnTe layer. The growth of $\mathrm{MnTe}$ layers was performed in Te rich conditions at the temperature of the substrate kept in the range $300-320^{\circ} \mathrm{C}$. In all cases a good quality, streaky RHEED (reflection high-energy electron diffraction) patterns were observed during the growth of MnTe layers, indicative of a two-dimensional growth. In most of the cases oscillations of RHEED intensity were also recorded enabling determination of the layers' thickness. The growth rate of MnTe layers was $0.4-0.7 \mu \mathrm{m} / \mathrm{h}$; the thickness of layers was in the range $0.5-5.6 \mu \mathrm{m}$.

\section{Results and discussion}

MnTe layers were characterized by: Nomarski contrast microscopy, X-ray analysis, Laue reflection patterns and photoluminescence (PL) measurements. The results of magnetic properties measurements are subject of a separate communication [6].

Nomarski contrast images showed that the MnTe layers grown on ZnTe buffer had the surface with some defects. The layers grown on CdTe buffer were highly reflective and shiny. Laue patterns of both types of layers had cubic symmetry and did not show any patterns corresponding to hexagonal symmetry.

The X-ray diffraction studies were performed using a single crystal diffractometer as well as a high resolution double-crystal diffractometer. The Bond method was applied for a precise lattice analysis. The lattice constant determined for the three layers grown in similar conditions was $(6.337 \pm 0.001) \AA$. This value is in agreement with earlier determinations [7]. The temperature coefficient of a lattice constant measured between $300 \mathrm{~K}$ and $500 \mathrm{~K}$ was $(5.0 \pm 0.2) \times 10^{-6} 1 / \mathrm{K}$. The variation of the lattice constant with temperature is shown in Fig. 1. The positions of various diffraction peaks originating in CdTe buffer and in MnTe layers did not reveal any disorientation between these layers. The width of the rocking curves (FWHM) of the buffers layers was measured to be about 10 arcmin whereas that of MnTe layers was 11-38 arcmin. The broadening of the peaks is mainly due to the mosaic structure as checked by an independent rotation of the sample and the counter. The evolution of FWHM of the rocking curves with the thickness of the layers grown in similar conditions showed no degradation of quality with the thickness. The best layer, $5.6 \mu \mathrm{m}$ thick with FWHM equal to $18 \mathrm{arcmin}$ is, probably, the thickest cubic MnTe specimen grown so far by MBE.

The photoluminescence spectra of several MnTe layers were measured. They are shown in Fig. 2. The luminescence is due to internal $\mathrm{Mn}^{2+}$ transitions. It occurs in the range of energies characteristic of this luminescence in bulk DMS at 


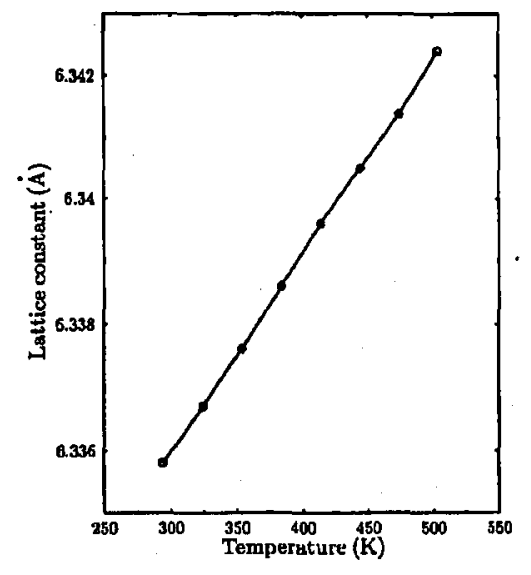

Fig. 1. Lattice constant as a function of temperature.

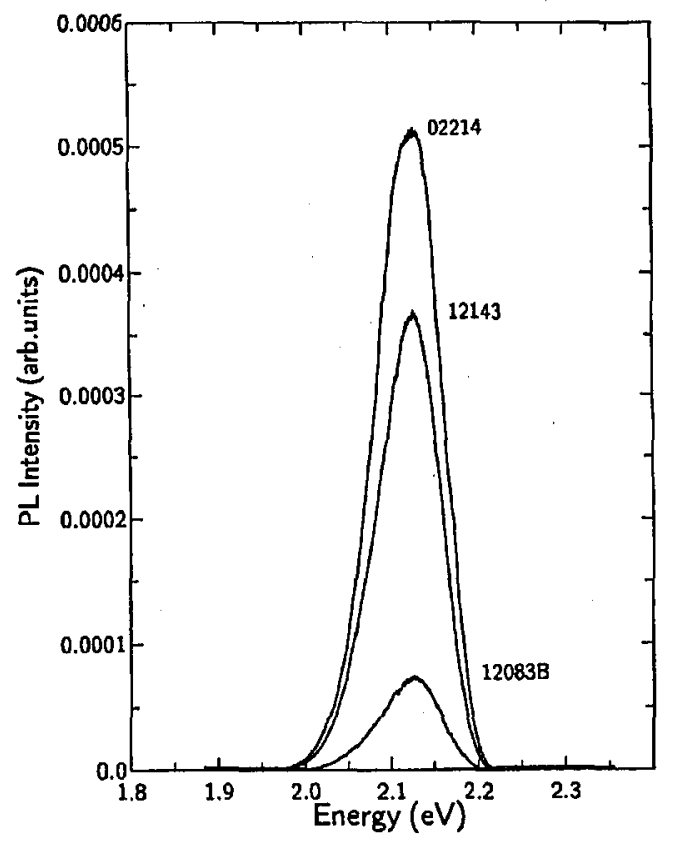

Fig. 2. Photoluminescence spectra of different MnTe layers at $10 \mathrm{~K}$. Sample $02214-$ $d=5.6 \mu \mathrm{m}, v_{\mathrm{gr}}=0.50 \mu \mathrm{m} / \mathrm{h}$, sample $12143-d=2.3 \mu \mathrm{m}, v_{\mathrm{gr}}=0.58 \mu \mathrm{m} / \mathrm{h}$, sample $12083-d=1.9 \mu \mathrm{m}, v_{\mathrm{gr}}=0.68 \mu \mathrm{m} / \mathrm{h}$.

a great dilution of Mn ions. This may be indicative of a substantial localization of the $d$ states also in "concentrated" pure MnTe. In the case of one of our layers the luminescence revealed a structure. The luminescence energy is shifted to shorter wavelengths compared to its position in MBE-grown $\mathrm{Cd}_{1-x} \mathrm{Mn}_{x} \mathrm{Te}, \mathrm{Zn}_{1-x} \mathrm{Mn}_{x} \mathrm{Te}$ 
layers for $x<1$. The temperature dependence of the luminescence energy was non-monotonic: it decreased slightly with temperature in the range from $10 \mathrm{~K}$ up to $70 \mathrm{~K}$, while above $70 \mathrm{~K}$ it increased with temperature. This type of behavior was not observed in the bulk DMS. Similar temperature dependence of $\mathbf{M n}^{2+}$ luminescence was observed in $\mathrm{Zn}_{1-x} \mathrm{Mn}_{x}$ Te layers [8].

\section{Conclusions}

Good quality thick epilayers of MnTe with zinc blende symmetry were grown by MBE. This structural quality persisted up to layer thicknesses as large as $5.6 \mu \mathrm{m}$. The value of the cubic lattice constant as well as its thermal expansion coefficient were determined. The luminescence due to internal $\mathrm{Mn}^{2+} d$-shell transitions was observed and found to exhibit non-monotonic temperature dependence.

The authors would like to acknowledge Professor A. Mycielski for supplying with high purity manganese. The study was partly supported by the grants PBZ-ZO11/P4/93/01 and 3 P407 00306 from the State Committee for Scientific Research (Republic of Poland).

\section{References}

[1] W.D. John Stone, A.E. Sestrich, J. Inorg. Nucl. Chem. 19, 229 (1961).

[2] W. Allen, G. Luckowsky, J.E. Mikkelsen, Solid State Commun. 24, 367 (1977).

[3] Semiconductors and Semimetals, Eds. J.K. Furdyna, J. Kossut, Vol. 25, Academic Press, New York 1987, Ch. 1.

[4] S.M. Durbin, J. Han Sungki O, M. Kobayashi, D.R. Menke, R.L. Gunshor, Q. Fu, N. Pelkanos, A.V. Nurmikko, D. Li, J. Gosalves, N. Otsuka, Appl. Phys. Lett. 55, 2087 (1989).

[5] K. Ando, K. Takahashi, T. Okuda, J. Magn. Magn. Mater. 104, 993 (1992).

[6] M. Sawicki, S. Koleśnik, T. Wojtowicz, G. Karczewski, E. Janik, M. Kutrowski, A.K. Zakrzewski, E. Dynowska, T. Dietl, J. Kossut, Acta Phys. Pol. A 87, 169 (1995).

[7] J.R. Bushert, F.C. Peiris, N. Samarth, H. Luo, J.K. Furdyna, Phys. Rev. B 49, 4619 (1994).

[8] M. Kutrowski, T. Wojtowicz, G. Karczewski, K. Kopalko, A.K. Zakrzewski, E. Janik, G.K. Grasza, E. Łusakowska, J. Kossut, Acta Phys. Pol. A 87, 500 (1995). 